\title{
Correction of MRI Image Intenseness by Minimization of Energy Approach
}

\author{
Navase Ali ${ }^{1}$ and Rakesh Kumar ${ }^{2}$ \\ Computer Science \& Engineering Department \\ National Institute of Technical Teachers Training and Research, \\ Chandigarh, India \\ navaselucky@gmail.com
}

\begin{abstract}
This paper presents the concept of correction of intensity inhomogeneity in MR images. The gradually shifting property of the inclination field got from the proposed vitality is actually guaranteed by the information term in our variety system, without the need to force an express smoothing term on the predisposition field. It assesses the execution of some chose MR pictures and furnishes the helpful outcomes with division and vitality advancement. In this, we apply level set technique for synchronous division and inclination rectification, particularly for MR pictures. The important parameter is entropy which is minimized up to 4.79 values by changing the knots. All simulations are done in MATLAB.
\end{abstract}

Keywords: MR images, Energy minimization, Image Processing, Spline Method, Optimization etc

\section{Introduction}

Human diseases diagnosis has been one of the principal goals in medicine. Since the beginning of the modern Medicine, all the research has been focused on finding the causes of the diseases and its cures by understanding human body behaviour. In the case of the neurological research this idea has been translated in finding the way of studying the brain in the harmless way. Since its first days MR technology has demonstrated to be the best and more reliable way to study inner tissues of human body specially the brain.

There is little uncertainty that imaging of the morphology and usefulness of the human body has upset social insurance. These days it is conceivable to analyse cells on an infinitesimal level, imagine their digestion system and even record a particular "unique finger impression" to distinguish them. Then again doctors can make morphologic 3-D pictures of the human body all in all and recognize the usefulness of censure and considerate tissues on a perceptible level. Past that imaging has expanded the nature of patient care as well as diminished the expenses for medicinal services. The purpose behind this is there are a great deal less superfluous strategies performed. Furthermore, numerous illnesses can be analysed in a substantially before phase of the movement since it is a bit much that side effects appear on the outside of the human body as it used to be.

$\mathrm{X}$-ray is an inestimable system that uses radio waves, intense magnet and a PC to identify detail pictures. Our body is comprised of a large number of hydrogen iota's (i.e.80\% of water) which are attractive in nature. At the point when our body is set in attractive field these particles adjust in the field much like a compass indicates the North Pole. A radio wave "thumps down" the particle and upsets their property. Attractive reverberation imaging depends on numerous physical properties of tissues and can

Received (May 25, 2017), Review Result (August 15, 2017), Accepted (August 24, 2017) 
accomplish perfect difference of delicate tissues with no radiation and in a totally nonintrusive way. The most well-known imaging techniques that have been utilized to picture atherosclerosis and stenosis depend on turn grid unwinding (T1), turn unwinding (T2), proton thickness (PD), dissemination, charge exchange (MT), stream (time-of-flight: TOF and stage differentiate: PC), and inclination reverberate (GRE). One of the main objectives of atherosclerosis imaging has been for quite a while to recognize and evaluate stenosis of vessels [1].

Three primary imaging modalities exist to evaluate stenosis: Ultrasound imaging, $\mathrm{X}$ beam imaging, and MRI. Early X-beam frameworks utilized a differentiation operator and planar projections to create diverse perspectives which, after understanding by a prepared master, delivered an estimation of the degree of lumen narrowing. Later systems utilize 3D CT (CTA), yet at the same time require the infusion of a difference operator obscure to X-beam. One preferred standpoint of CTA is the unmistakable perception of calcifications and solidifications, quite often related to cutting edge atherosclerotic injuries [2].

X-ray shows the upside of permitting synchronous representation of the blood vessel lumen and vessel divider, and further can possibly describe atherosclerotic plaques. Real plaque segments, for example, the lipid center, calcification, stringy connective tissue, and intra-plaque drain/thrombus have been related to respect to their flag force qualities on dark blood (where blood flag is scratched off and the lumen shows up in this manner dark) T1W, T2W and PDW pictures. These dark blood MRI methods are additionally helpful for vessel divider range estimation, a for the most part acknowledged direct measure of plaque weight.

The paper is organized as follows. Section II presents the related work of system. In section III, it describes concept of MR images. In Section IV, it describes the description of proposed system used in processing technique. The results are given in Section V. Finally, conclusion is explained in Section VI.

\section{Related Work}

P. F. Alcantarilla et al. (2011) introduced a successful framework for distinguishing vehicles before a camera-helped vehicle (going before vehicles going in a similar course and approaching vehicles going the other way) amid evening driving conditions so as to naturally change vehicle head lights between low bars and high bars staying away from glares for the drivers. Moreover, low bars yield was chosen when the vehicle was in a sufficiently bright or urban range. Picture preparing systems were connected to dissect light sources and to recognize vehicles in the pictures. The calculation time spent on handling one info casing was relying upon its street scene intricacy and the quantity of blobs [3].

V. Caselles (2011) introduced that the in painting issue can be detailed in a measurable mechanics structure, in which amplification of the self-similitude of the remade picture with a uniform earlier to select similarly comparable patches, communicated as an augmentation of the entropy. Picture in painting otherwise called "picture consummation" or "disocclusion" is the recuperation of absent or adulterated parts of a picture in a given district so that the remade picture looks characteristic. This general detailing shrouded in painting and in addition picture reproduction from an arrangement of meagre specimens, in which case tempering was suggested. The initial phase in the process was the estimation of the profundity of the scene and its recreation in the districts to be inpainted [4].

Y. Zheng et. al. (2012) displayed novel procedures for single-picture vignetting redress in light of symmetries of two types of picture inclinations: half circle distracting slopes (SCTG) and outspread angles (RG). For a given picture pixel, a SCTG is a picture slope along the distracting course of a hover focused at the assumed optical focus and going 
through the pixel. They watched that the symmetry properties of SCTG and RG appropriations are firmly identified with the vignetting in the picture. In light of these symmetry properties they built up a programmed optical focus estimation calculation by limiting the asymmetry of SCTG circulations, and furthermore exhibited two strategies for vignetting estimation in view of limiting the asymmetry of RG appropriations [5].

D. Baswaraj et. al. (2012) introduced the scientific categorization and the present best in class in Image division and utilization of Active Contours. Dynamic shapes have been broadly utilized as appealing picture division techniques since they generally deliver subareas with consistent limits. The utilization of level set hypothesis has given greater adaptability and comfort in the usage of dynamic shapes. Dynamic form models (ACMs) incorporated with different sorts of outer compel fields to pull the shapes to the correct limits have demonstrated their capable capacities in protest division [6].

$\mathrm{X}$. Hou et. al. (2012) presented a basic picture descriptor alluded to as the picture signature. They appeared, inside the hypothetical structure of inadequate flag blending, that this amount spatially approximates the forefront of a picture. They tentatively explored whether this rough forefront covers with outwardly obvious picture areas by building up a saliency calculation in view of the picture signature. This saliency calculation predicts human obsession focuses best among contenders on the Bruce and Tsotsos benchmark informational collection and does as such in significantly shorter running time. In a related analysis, they exhibited with a change visual deficiency informational collection that the separation between pictures prompted by the picture mark is nearer to human perceptual separation than can be accomplished utilizing other saliency calculations [7].

W. Li et. al. (2013) displayed a novel non-unbending optical stream calculation for thick picture correspondence and non-inflexible enrollment. The calculation utilized an extraordinary Laplacian Mesh Energy term to energize neighborhood smoothness while at the same time protecting non-inflexible disfigurement. Laplacian distortion approaches had turned out to be prominent in design inquire about as they empower work disfigurements to safeguard neighborhood surface shape. In this work they proposed a novel Laplacian Mesh Energy equation to guarantee such sensible nearby distortions between picture sets. They communicated this entirely inside the optical stream enhancement, and demonstrate its application in a novel coarse-to-fine pyramidal approach [8].

S. Konduri et. al. (2014) recommended that MRI power inhomogeneities could be ascribes to defects in the RF. The outcome was gradually changing shading ancient rarity over the picture that could create the blunders. Accordingly by limiting this vitality the level set technique could at the same time fragment the picture and gauge the predisposition field, which could be utilized for inclination rectification. A variety level set structure for division and predisposition revision of pictures with power inhomogeneities. In this work neighbourhood picture data was used productively and thusly could at the same time section and inclination rectify the pictures with force inhomogeneity. Factual examination was performed by computing likelihood, change and entropy for both the pictures [9].

Archana et. al. (2014) proposed a novel locale based strategy for picture division, which was managed force inhomogeneity pictures. Initially in light of a model of pictures with force inhomogeneities, they determined a nearby power grouping property of the picture powers and characterize a neighbourhood bunching rule work for the pictures powers in an area of each point. This nearby bunching standard was then incorporated as for the area focus to give worldwide basis of picture division. In a level set definition, this rule characterized a vitality as far as level set capacity that speak to a parcel of the picture space and inclination field that records for the force inhomogeneity of the picture [10].

S. Mukherjee et. al. (2015) proposed a novel area based division strategy equipped for fragmenting objects in nearness of huge power variety. Current arrangements utilized 
some type of nearby preparing to handle intra-area inhomogeneity, which made such strategies helpless to neighbourhood minima. In this work, they introduced a structure which sums up the customary Chan-Vese calculation. Rather than existing nearby methods, they spoke to the light of the areas of enthusiasm for a lower dimensional subspace utilizing an arrangement of pre-determined premise capacities [11].

K. Zhang et. al. (2016) displayed a novel level set technique for picture division within the sight of force inhomogeneity. The inhomogeneous articles were demonstrated as Gaussian conveyances of various means and differences in which a sliding window was utilized to outline unique picture into another area, where the force dissemination of each protest was still Gaussian however better isolated. The method for the Gaussian disseminations in the changed area can be adaptively evaluated by increasing an inclination field with the first flag inside the window [12].

\section{Description of MRI Images}

MRI is a priceless procedure that utilizes radio waves powerful magnet and a computer to detect detail images. Our body is made up of millions of hydrogen atoms (i.e.80\% of water) which are magnetic in nature. When our body is placed in magnetic field these atoms align in the field, much like a compass point to the North Pole. A radio wave "knocks down" the atom and disrupts their property. Magnetic resonance imaging is based on many physical properties of tissues and can achieve exquisite contrast of soft tissues, without any radiation and in a completely non-invasive way. The most common imaging methods that have been used to image atherosclerosis and stenosis are based on spin-lattice relaxation (T1), spin-spin relaxation (T2), proton density (PD), diffusion, magnetization transfer (MT) [13], flow (time-of-flight: TOF and phase contrast: PC), and gradient echo (GRE)

Three main imaging modalities exist to quantify stenosis: Ultrasound imaging, $\mathrm{X}$ ray imaging, and MRI. Early X-ray systems used a contrast agent and planar projections to generate different views which, after interpretation by a trained expert, produced an estimation of the extent of lumen narrowing. More recent techniques use 3D CT (CTA) [14] but still require the injection of a contrast agent opaque to X-ray. One advantage of CTA is the clear visualization of calcifications and ossifications, almost always associated to advanced atherosclerotic lesions [15]. The major drawback of X-ray modality is the use of ionizing radiations. This problem can be avoided by using magnetic resonance angiography (MRA). MRI is safe and allows imaging of the vasculature either with a contrast agent, or in absence of a contrast agent owing to the flowing properties of the blood.

MRI presents the advantage of allowing simultaneous visualization of the arterial lumen and vessel wall, and further has the potential to characterize atherosclerotic plaques. Figure 1 shows major plaque components, such as the lipid core, calcification, fibrous connective tissue, and intra-plaque hemorrhage/thrombus have been identified with regard to their signal intensity characteristics on black blood (where blood signal is canceled and the lumen appears thus black) T1W, T2W and PDW images. These black blood MRI techniques are also useful for vessel wall area measurement, a generallyaccepted direct measure of plaque burden.

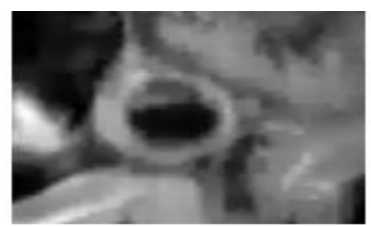

PDW

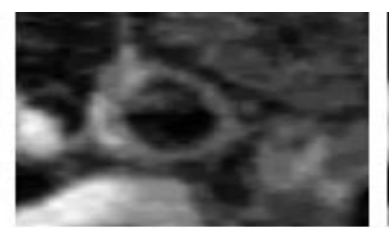

T2W

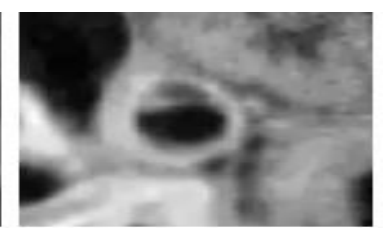

T1W

Figure 1. Tissue Classification [16] 
Two major contrast agents are used in MRI: Gadolinium-based molecules that decrease T1 relaxation times, and small iron oxide particles that disturb the magnetic field. The former has a diameter of about $0.8 \mathrm{~nm}$ for the common Gd-DTPA. The latter has many forms that span diameters from $4 \mathrm{~nm}$ to $1000 \mathrm{~nm}$ or bigger. SPIO have a typical size of 70-140 nm. For comparison Albumin has a diameter of about $7 \mathrm{~nm}$ and a size of 68 kilo dalton. Gd-DTPA contrast agents are small molecules that can diffuse through the endothelium over time, whereas the bigger iron oxide particles require a much longer time to do so. In order to get a significant sensitivity with MRI with a CA, a concentration of 1 $\mathrm{mm}$ is necessary. This concentration is obtained after 1 hour when particle size is about $10 \mathrm{~nm}$. Therefore, these MRI [16] contrast agents will not penetrate cells nor diffuse significantly through the normal endothelium barrier in the time compatible with clinical imaging.

So, from above details, multi-contrast MRI [17] can characterize atherosclerotic lesions in vivo. Beyond expert examination of multiple images taken at the same location, whether they are regular spin-echo acquisitions, more advanced sequences like magnetization transfer, or contrast-enhanced data set using recent molecular imaging advances, computer methods are promising to reduce substantially the time and therefore the cost of analyzing such big data sets by providing the physicians with a reliable and accurate meta information display of the underlying disease.

\section{Improve Quality of MR Images by Energy Optimization}

A basic stride for examination strategies depending on voxel dim qualities is the rectification of the flag forces over the MR picture. The primary wellspring of the debasement is the spatial inhomogeneity of curl affectability of the extraordinarily outlined surface loops. A redress calculation for carotid vein imaging faces many difficulties. To start with, the get curls experience the ill effects of an exceptionally soak affectability tumble off toward expanding tissue profundity that is a great deal more noteworthy than the variety over the mind when imaging with a head loop. If not all around rectified, this can frustrate the examination of the vessel divider by specialists and annihilation programmed tissue characterization calculations. Second, the clamor exhibit in the MRI carotid pictures can disturb calculations. Third, there are numerous voxels near the course dividers that are bereft of flag, either from fat concealment or from blood stream pay. Such voxels don't give data about the predisposition field [18].

It proposes a technique that expands upon work in the writing and addresses issues with the carotid supply route pictures. To maintain a strategic distance from the disadvantages of order plans, we limit entropy. To stay away from the debasement from low SNR districts, we advance entropy locally, beginning with high SNR territories and blending ranges with lower SNR in a successive manner. Picture handling is utilized to naturally recognize all the tissue voxels in the neck. To start with, we will recognize all the air voxels outside the neck.

MRI resolution can be decreased but at the expense of time or noise. Limited resolution has two consequences. First, sharp edges between two distinct tissues are blurred (e.g., lumen/vessel interface). Second, anatomical structures smaller than the voxel size disappear (e.g., small calcification inside the lipid core). This problem is exacerbated by the noise: when a voxel is slightly brighter than its surrounding voxels. We tried a new approach to this problem based on non-linear diffusion filtering that can reduce partial volume effect when it is possible, without generating artifacts or spurious features when it is not. We have also extended the technique by incorporating a noise reduction term.

After reading the required input image, it first requires the segmentation of particular portion. Level Set Method is one of the emerging image segmentation techniques for medical image segmentation. The level set method is a numerical technique for tracking interfaces and shapes. It was first introduced by Osher and Sethian to capture moving 
fronts in 1987. The basic idea of the level set method is to represent contours as the zero level set of an implicit function defined in a higher dimension, usually referred to as the level set function, and to evolve the level set function according to a partial differential equation (PDE). In typical PDE methods, images are assumed to be continuous functions sampled on a grid. Active contours were introduced in order to segment objects in images using dynamic curves.

We first identify all tissue voxels and filter the image to reduce noise. We fit a third order polynomial function to the tissue voxels so as to provide a rough initial estimate of the bias field, B0. Air voxels in the background are excluded because they are void of signal. For the refined description, we model the bias field, B, as a spline with a rectangular grid of knots evenly spaced across the image.

The cluster values to be optimized, and it is a non-negative weighting function such that $\mathrm{R}$ and $\mathrm{Lx}=1 \mathrm{r}$ is the radius of $\mathrm{Ly}$. In the method, the weighting function is chosen as the Gaussian kernel. The ultimate goal is to find an optimal set of partitions for the entire image domain $\mathrm{O}$, the bias field $\mathrm{B}$, and the constants ci. The minimization of a single objective function for a point y does not accomplish this goal. The method minimizes for the entire points y. This can be achieved by minimizing the integral of over $\mathrm{O}$. In this work, we consider the case of $\mathrm{N}=2$, the multi-phase case can be solved with a similar procedure. In this case, the image domain is partitioned into two regions corresponding to the object and background. We assume that these two regions can be represented by the regions separated by the zero level contour of function $\mathrm{f}$. In numerical implementation each iteration, the variables are updated such that for fixed $\mathrm{f}$ and $\mathrm{B}$, we find an optimal solution that minimizes Energy.

We now describe the piecewise optimization process which makes the use of a bicubic spline model tractable. We identify the knot k1having the highest corresponding B0 value and begin optimization there. The signal from the coil at this location is high and the high local SNR ensures that we will get a good local estimate of B.

\subsection{Level Set Segmentation}

In this situation, the proposed level set function will develop steep or flat gradients leading to problems in numerical approximations. In existing work the shape changing its topology by splitting in two. It would be quite hard to describe this transformation numerically by parameterizing the boundary of the shape and following its evolution. In $2-\mathrm{D}$, it is represented by

$$
\Gamma=\{(x, y) \mid \psi(x, y)=0\}
$$

It is then necessary to reshape the level set function to a more useful form, while keeping the zero location unchanged. A technique for periodically re-initializing the level set function to a signed distance function is presented in this work. It consists of an internal energy term that penalizes deviations of the level set function from a signed distance function, and an external energy term that drives the motion of the zero level set toward the desired image feature. Second, a fuzzy membership mask image, M, is created with a label of one for tissue voxels, zero for signal voids, and a value between zero and one for partial volume voxels. Third, the fuzzy membership mask $\mathrm{M}$ is cleaned so as to remove incorrectly identified background voxels. $M$ is thresholded to yield a binary mask for $\mathbf{M}>0$. The resulting binary image is processed using opening to remove small islands of pixels and closing to remove small holes. It helps to provide better results than existing one.

\subsection{Noise Reduction Filtering}

The image is subject to a diffusion process perpendicular to the gradient when it is lower than $\sigma_{\mathrm{AD}}$ and is unchanged otherwise. It used the estimated noise variance from the background voxels to obtain the parameter $\sigma_{\mathrm{AD}}$. Assuming that the noise, n,is stationary, 
Gaussian, and independent with standard deviation $\sigma_{\mathrm{n}}$, then $\nabla x$ is also normally distributed with zero mean and standard deviation $\sqrt{2}$.We set the number of iterations of the anisotropic diffusion filtering to nine voxels by analogy to linear Gaussian filtering

\subsection{Bias Correction}

The polynomial function is fitted in a least square sense to the tissue pixels using a classic regression technique. To improve upon the description of the bias field, we use a bicubic spline model. In the method, the weighting function is chosen as the Gaussian kernel. The ultimate goal is to find an optimal set of partitions for the entire image domain $\mathrm{O}$, the bias field $\mathrm{B}$, and the constants ci. The minimization of a single objective function for a point $y$ does not accomplish this goal. The method minimizes for the entire points $y$. This can be achieved by minimizing the integral of over O. To initialize this estimate, we take the value of $\mathrm{B}_{0}$ at the knot locations. After each optimization step, we divide the bias field $\mathrm{B}$ by a constant so as to ensure that it is mean over the tissue voxels is 0.5 .

\subsection{Energy Minimization}

Let $\mathrm{B}(\mathrm{x})$ be an unknown bias field, $\mathrm{J}(\mathrm{x})$ be the true signal to be restored, and $\mathrm{N}(\mathrm{x})$ be noise. We consider the following model of intensity inhomogeneity

$$
I(x)=B(x) J(x)+N(x)
$$

Noise $\mathrm{N}(\mathrm{x})$ is assumed to be Gaussian-distributed with zero mean. We define a mapping from original image intensity domain to another domain by averaging image intensities:

$$
I(x)=\frac{1}{L(x)} \int I(y, B, x) d y
$$

In existing work, the energy functional is represented by:

$$
E=\frac{\pi \rho^{2}}{2} \sum \int(I(y)-c)^{2} M(\varphi(y)) d y
$$

Where $\mathrm{M}$ is phase indicator, $\varphi$ is level set function, $\mathrm{I}(\mathrm{y})$ is intensity of image and $\mathrm{c}$ is constant function. The bicubic spline estimate of the bias field is optimized so as to minimize the entropy of the image using the piecewise, optimize and merge algorithm described previously.

$$
x(t)=\sum P i N i^{d}(t)
$$

$\mathrm{d}=$ degree of curve with knot sequence $(0 \ldots . \mathrm{n})$ and $\mathrm{N}$ is basis function. Using B-Spline Model, the energy is represented by:

$$
\begin{gathered}
E(x)=\int|x(t)|^{2} d t \\
=\sum \sum P i P j \int N i^{d}(t) N j^{d}(t) d t
\end{gathered}
$$

In this work, we consider the case of $\mathrm{N}=2$, the multi-phase case can be solved with a similar procedure. 


\section{Proposed Algorithm of System}

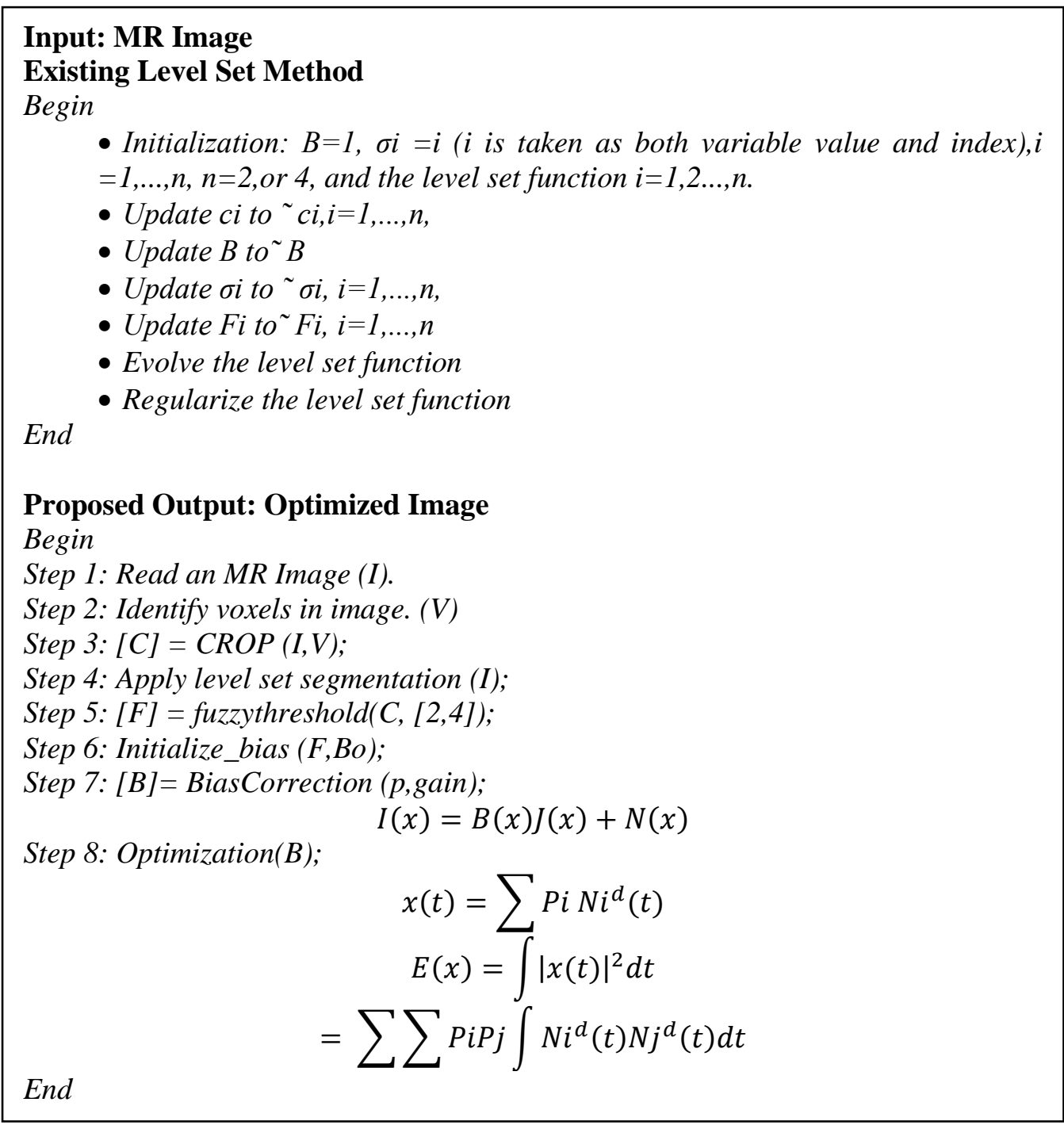

\section{Results of Proposed System}

In this work, a level set segmentation method is used in MR images and then energy optimization concept is carried out. MR outputs were gotten of a homogeneous, salinefilled, round and hollow apparition about the span of a human neck. These pictures were procured utilizing the staged cluster surface curls and the subsequent picture, in the wake of normalizing the most extreme incentive to one, gave the genuine inclination field. We first identify all tissue voxels and filter the image to reduce noise. We fit a fourth-order polynomial function to the tissue voxels so as to provide a rough initial estimate of the bias field. Air voxels in the background are excluded because they are void of signal. For the refined description, we model the bias field, as a bicubic spline with a rectangular grid of knots evenly spaced across the image as shown in Figure $2 \& 3$. 


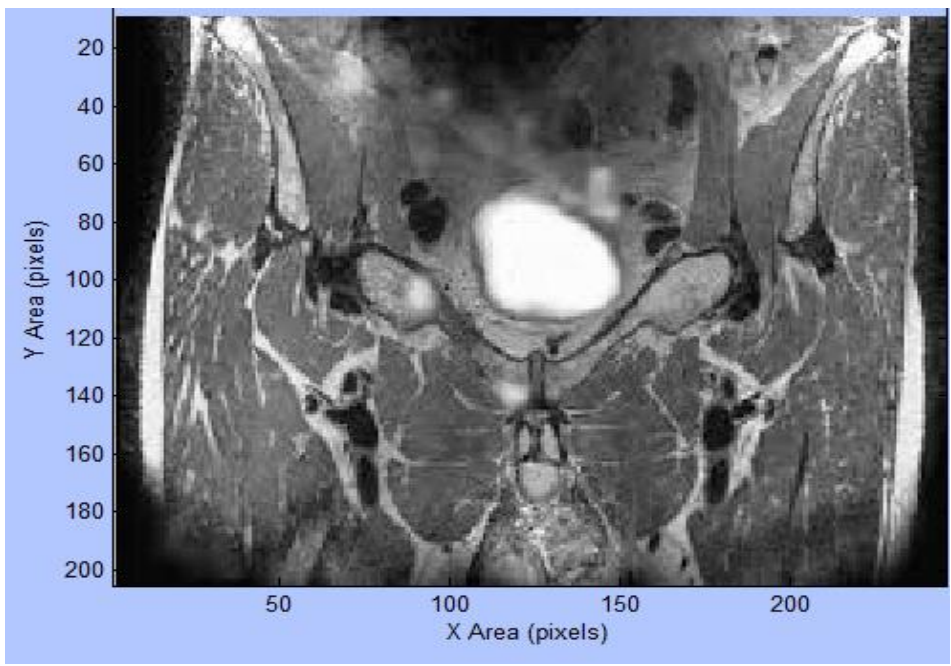

Figure 2. Input MRI Image

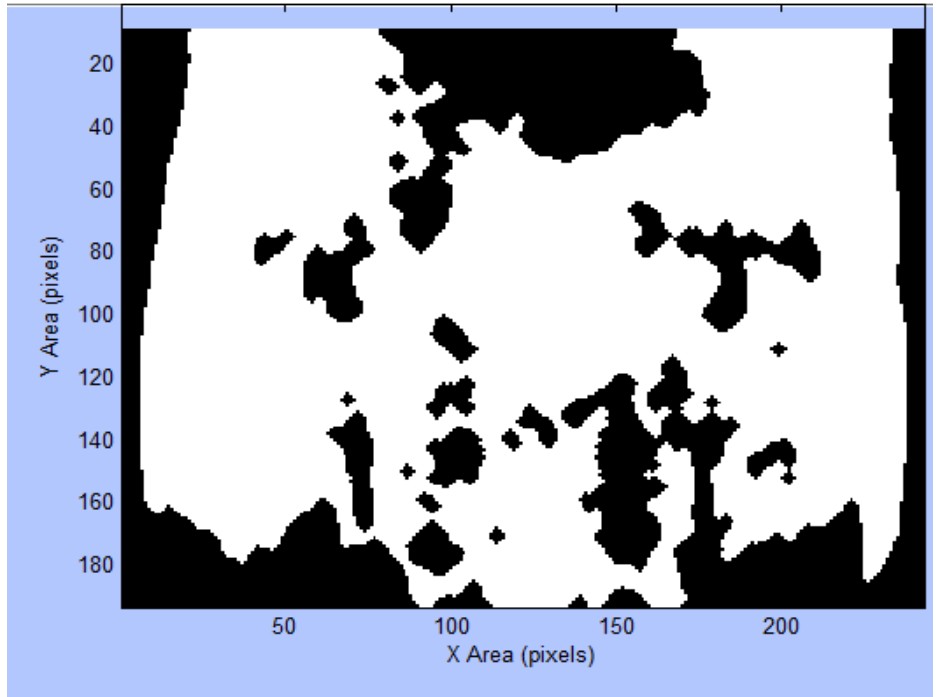

Figure 3. Image Segmentation

The spacing of knots is important: knots should be sufficiently close to ensure that the bias field can be adequately expressed and far enough apart that the estimated surface will not contain anatomical structures in the images. Spacing is related to the receiver coil geometry and is optimized in experiments described later. We use the values of the initial polynomial estimate of the bias field at the knot locations to initialize the bicubic spline bias field. We now describe the piecewise optimization process which makes the use of a bicubic spline model tractable. We identify the knot having the highest corresponding value and begin optimization there. The signal from the coil at this location is high and the high local SNR ensures that we will get a good local estimate of B.

In this case, the image domain is partitioned into two regions corresponding to the object and background. We assume that these two regions can be represented by the regions separated by the zero level contour of function $\mathrm{f}$. In numerical implementation each iteration, the variables are updated such that for fixed $\mathrm{f}$ and $\mathrm{B}$, we find an optimal solution that minimizes Energy. The object and background have the same intensity means but different variances. It can be seen that the model, which assumes that an image consists of statistically homogeneous regions fails to extract the object boundary as shown in Figure 4 and 5 respectively. 


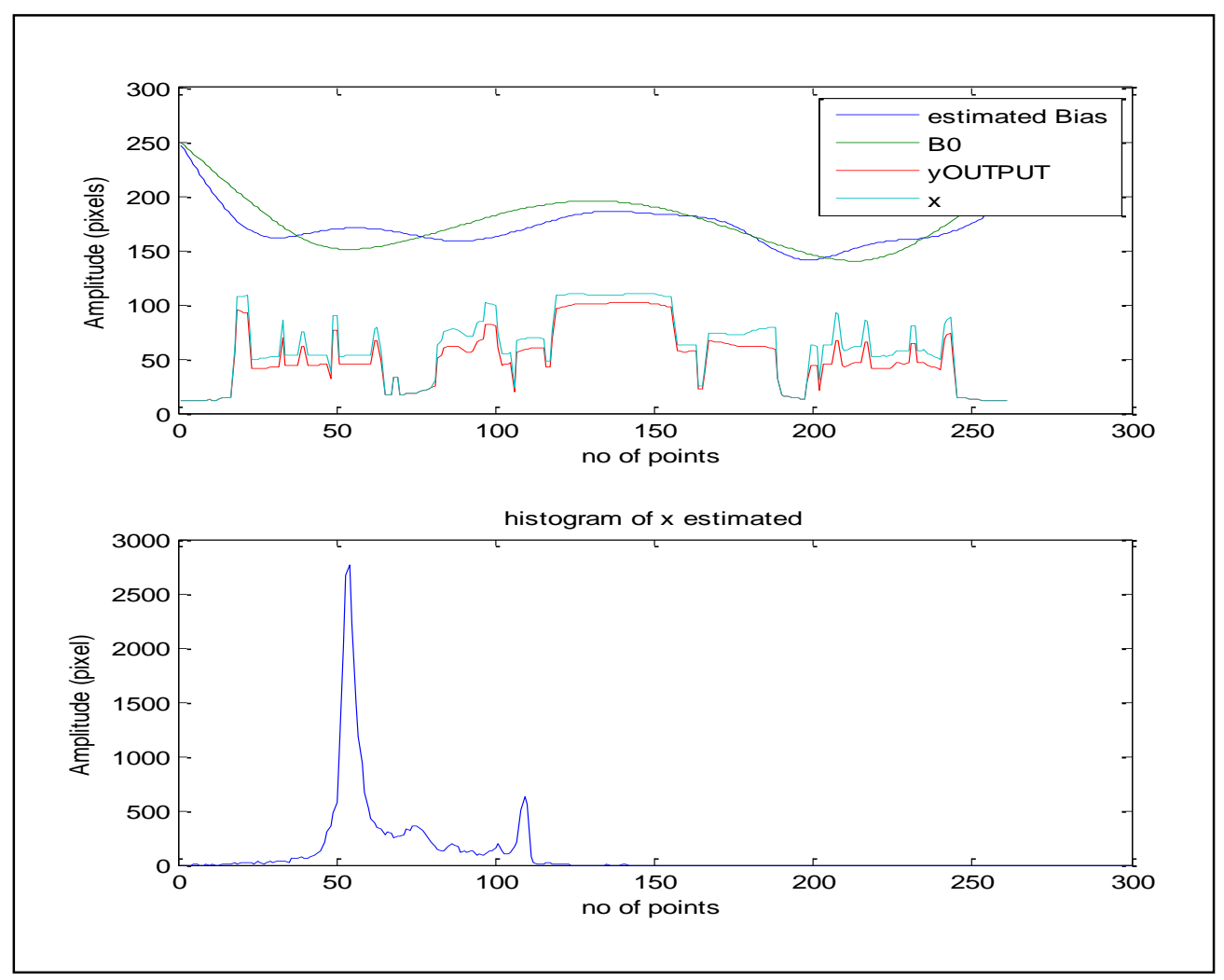

Figure 4. Bias Estimation and Its Histogram

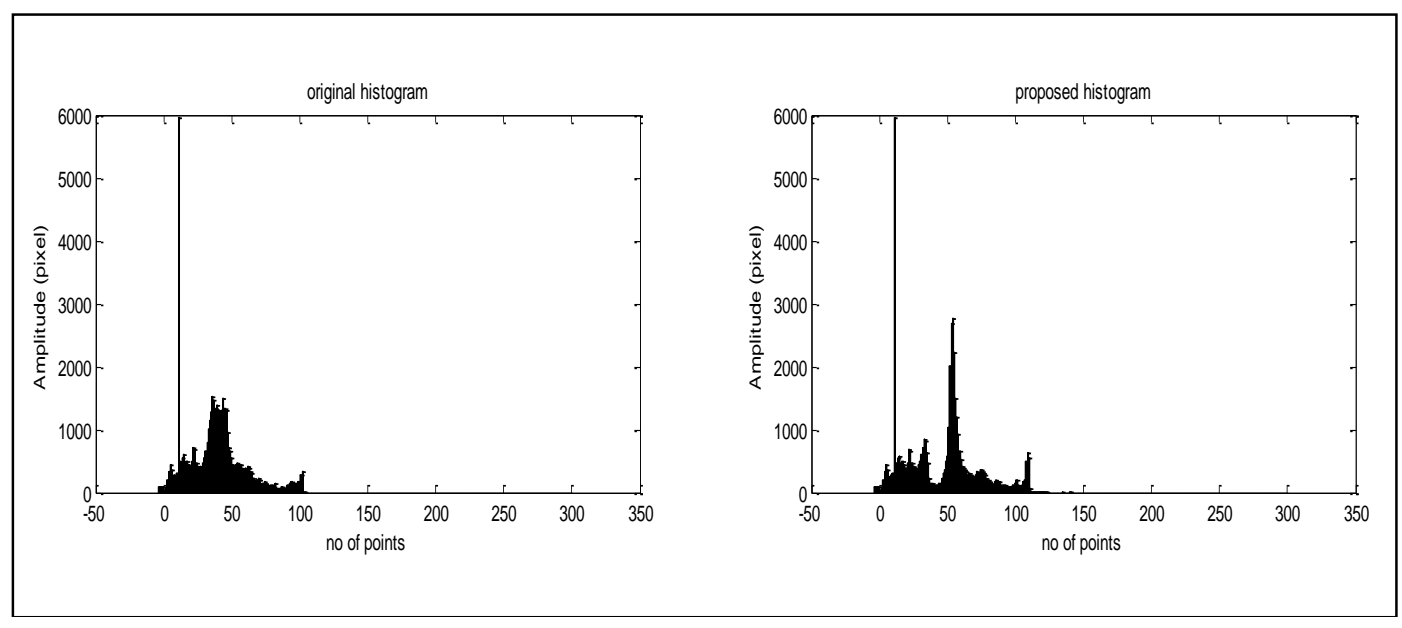

Figure 5. Original and Proposed Histogram of Image

In previous work, there is problem in segmentation that does not include side lines and does not provide energy minimization upto a certain extent because of two phase method used. We described a new method to correct for intensity inhomogeneity of images. By optimizing a cubic spline to minimize the entropy of a dataset, a bias field can be estimated and the images corrected. Many methods exist to address this problem but it did not provide good results in the case of strong inhomogeneity. In segmentation of images, the proposed results show better segmented output as compared to actual one. We can observe that segmentation results are visually satisfying although the noises are very strong, thereby validating the robust performance of our method to Gaussian noise. The results are shown in Figure 6 and performance comparison of proposed system with existing one is shown table 1 . Due to this, after energy minimization, the histogram shows 
better spikes and area is distributed uniformly as compared to actual one. The improvement of the image quality in terms of intensity homogeneity can be demonstrated by visualizing the histograms of the original images and the bias corrected images. The histograms of the original images (left) and the bias corrected images (right) are plotted.

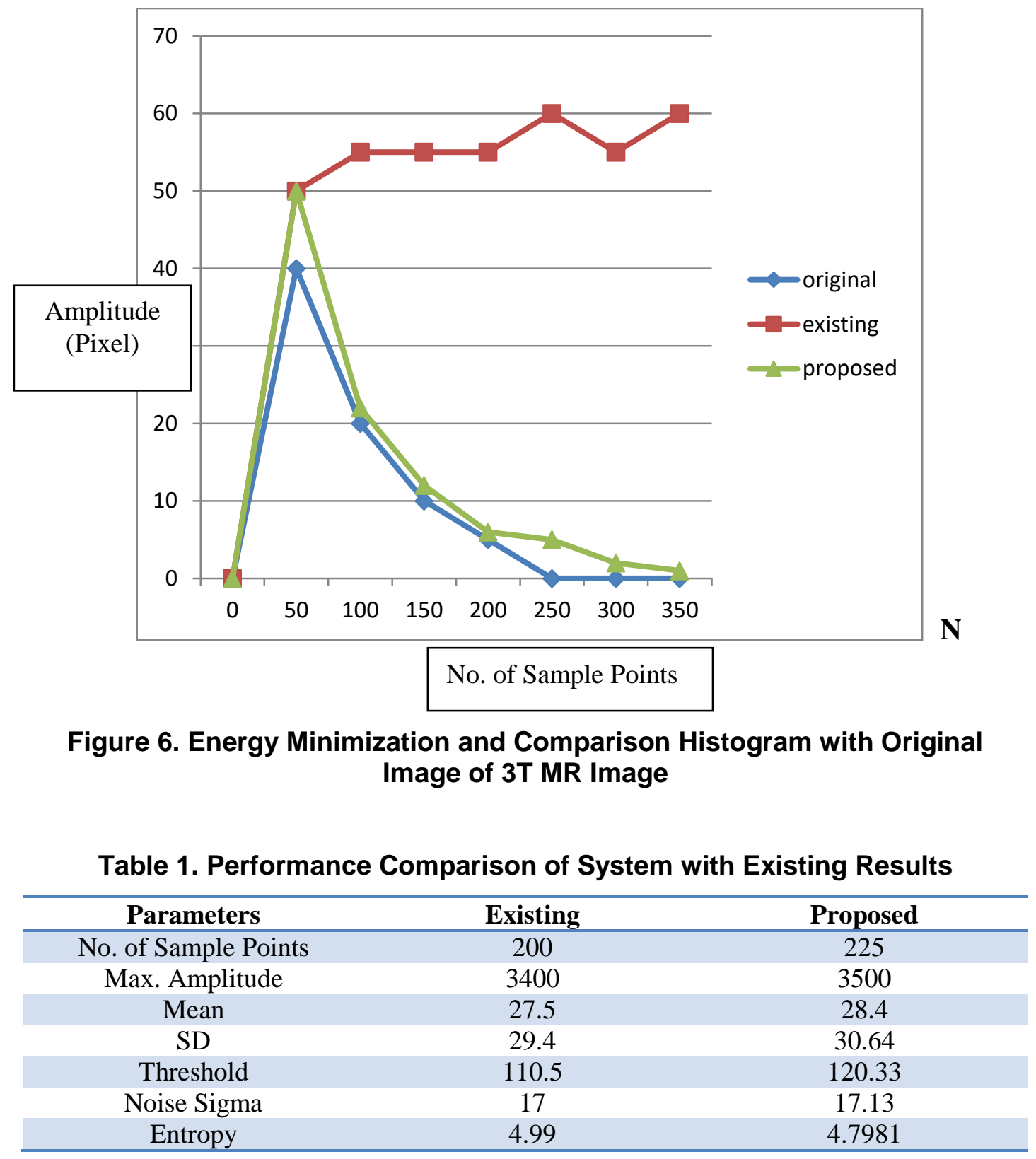

\section{Conclusion}

In this work, it displayed a variational level set structure for division and predisposition remedy of pictures with force inhomogeneities. In light of a for the most part acknowledged model of pictures with power inhomogeneities and a determined neighbourhood force grouping property, we characterize vitality of the level set capacities that speak to a segment of the picture space and an inclination field that records for the force inhomogeneity. Division and inclination field estimation are subsequently mutually performed by limiting the proposed vitality useful. We portrayed another technique called vitality minimization to adjust for force inhomogeneity of pictures. By upgrading a cubic 
spline to limit the entropy of a dataset, a predisposition field can be evaluated and the pictures amended. The proposed technique was intended for those troublesome cases and demonstrated great outcomes for surface loops, additionally for interventional MRI, presumably the most outrageous instance of inhomogeneity.

In future, for improvement of tracking, elements other than the distance can be added to the benefit function. The use of global and local S-curve can performs better and shows efficiency for the future development of methods for segmentation.

\section{References}

[1] R.C. Gonzalez and R. Woods, "Digital Image Processing”, 2nd Edition, Prentice-Hall, (2002).

[2] R. Gonzalez, R. Woods and S. Eddins, "Digital Image Processing Using Matlab", Prentice Hall, (2004).

[3] P. F. Alcantarilla and L. M. Bergasa, "Automatic Light Beam Controller for driver assistance", Machine Vision and Applications, Springer-Verlag, vol. 22, Issue 5, (2011), pp. 819-83.

[4] V. Caselles, "Exemplar-based Image Inpainting and Applications", International Council for Industrial and Applied Mathematics, vol. 44, no.10, (2011).

[5] Y. Zheng and S. Lin, "Single-Image Vignetting Correction from Gradient Distribution Symmetries", IEEE Transactions on Pattern Analysis and Machine Intelligence, vol. 35, no. 6, (2012), pp. 1480-1495.

[6] D. Baswaraj and A. Govardhan, "Active Contours and Image Segmentation: The Current State of the Art", Global Journal of Computer Science and Technology Graphics \& Vision, vol. 12, Issue 11, Version I, (2012), pp.1-12.

[7] X. Hou and J. Harel, "Image Signature: Highlighting Sparse Salient Regions", IEEE Transactions on Pattern Analysis and Machine Intelligence, vol. 34, no. 1, (2012), pp.194-201.

[8] W. Li, "Optical Flow Estimation using Laplacian Mesh Energy", IEEE Computer Vision Foundation, (2013), pp. 2435-2444.

[9] S. Konduri and A. Thirupathaiah, "A level set Image Articulations in presence of disagreement with MRI Scanning”, International Journal of Research in Computer and Communication Technology, vol. 3 , Issue 1, (2014), pp. 1-4.

[10] R. P. Archana, "Medical Application of Image Segmentation with Intensity Inhomogeneities", International Journal of Scientific Engineering and Research (IJSER), vol. 2, Issue 3, (2014), pp. 62-65.

[11] S. Mukherjee and S. T. Acton, "Region Based Segmentation in Presence of Intensity Inhomogeneity Using Legendre Polynomials", IEEE Signal Processing Letters, vol. 22, no. 3, (2015),pp. 298-302.

[12] K. Zhang and L. Zhang, "A Level Set Approach to Image Segmentation with Intensity Inhomogeneity", IEEE Transactions on Cybernetics, vol. 46, no. 2, (2016), pp. 546-557.

[13] M. Nikolova and M. K. Ng, "Fast Nonconvex Nonsmooth Minimization Methods for Image Restoration and Reconstruction", IEEE Transactions on Image Processing, vol. 19, Issue 12, (2010), pp. 30783088 .

[14] K. Bhima and A. Jagan, "An Efficient Method for Identification of Anomalies in Brain MR Images", IEEE Signal Processing Letters, vol. 10, no. 3, (2016), pp. 1-5.

[15] A. Gandhamal, S. Talbar and S. Gajre, "A Generalized Contrast Enhancement Approach for Knee MR Images", IEEE International Conference on Signal and Information Processing, (2016), pp. 1-6.

[16] S. Lu, L. Lei and H. Huang, "A Hybrid Extraction-Classification Method for Brain Segmentation in MR Image", IEEE International Congress on Image and Signal Processing, Bio-Medical Engineering and Informatics, (2016), pp. 1381-1385.

[17] S. Samant, P. K. Nanda and A. Ghosh, "Noisy Brain MR and CT Image Registration using MRF model", IEEE Region 10 Conference, (2016), pp. 1258-1261.

[18] Y. Han, H. Du, X. Gao and W. Mei, "MR Image Reconstruction Using Co-support Constraints and Group Sparsity Regularization”, IET Image Processing, vol. 11, Issue 3, (2017), pp. 155-16. 